\title{
Assessment of variable step-size integration of multibody systems
}

\author{
Maurizio Ruggiu ${ }^{1}$, Francisco González ${ }^{2}$ \\ ${ }^{1}$ Dipartimento di Ingegneria Meccanica, \\ Chimica e dei Materiali \\ Università di Cagliari \\ Via Marengo, 2, 09123 Cagliari, Italy \\ maurizio.ruggiu@dimcm.unica.it \\ ${ }^{2}$ Laboratorio de Ingeniería Mecánica \\ University of A Coruña \\ Mendizábal s/n, 15403 Ferrol, Spain \\ f.gonzalez@udc.es
}

\begin{abstract}
When performing the numerical integration of multibody systems (MBS) dynamics, the analyst can choose from a wide variety of methods and implementations. Selecting the most appropriate option for a particular application is not a straightforward task; as a consequence, several benchmark examples have been formulated by the MBS research community with the intent to assess the accuracy and performance of different solution methods when applied to certain kinds of mechanical problems. This paper introduces a variation of the slider-crank mechanism, already employed as benchmark problem in the MBS literature, intended to evaluate the performance of variable-step MBS algorithms. Three cases, featuring singular configurations and variable-frequency external actions, were defined. The example is used to illustrate some necessary elements in the definition of a benchmark problem and in the process of comparing different solution methods, as well as difficulties that can arise during this task. The proposed example was used to evaluate the behaviour of a variable-step index-3 augmented Lagrangian algorithm with velocity and acceleration projections, as well as other well-known solution methods.
\end{abstract}

Keywords: Benchmark problems, Numerical integration, Efficient Methods, Singular configurations.

\section{INTRODUCTION}

A considerable number of methods and algorithms for the simulation and analysis of Multibody System (MBS) Dynamics have been proposed since the early developments in this area were first published $[1,2]$. The performance of each approach depends on the characteristics of the problems to which it is applied, and so methods that are effective in the simulation of a certain type of mechanical system may be inefficient when applied to mechanisms with a different topology or subjected to other kinds of physical phenomena. Fully recursive methods [3], for instance, may become ineffective in the solution of heavily constrained multibody systems; mechanisms that feature redundant constraints or singular configurations pose a problem for solution algorithms that expect the Jacobian matrix of the constraints to have a full row rank throughout the motion $[4,5]$. Moreover, implementation techniques, third-party software libraries, e.g., for the linear algebra routines required by most MBS codes, and the hardware platform used to execute the code, as well as the interaction between them, have a critical impact on the time elapsed in computations $[6,7]$. For these reasons, selecting an appropriate MBS formalism for its application to a particular problem may prove challenging in some cases, particularly when efficiency constraints are imposed as a requirement.

Benchmark problems represent a useful tool to evaluate the accuracy and efficiency of MBS codes, as well as their ability to handle particular kinds of problems. Ideally, benchmarks should be simple enough to enable their exact reproduction by any researcher or team interested in using them. At the same time, they must be nontrivial problems that provide interesting information about some aspect of the behaviour of the solution method [8]. In recent years, several initiatives have been put 
forward by MBS researchers to propose meaningful test problems that can be generally accepted as benchmarks by the community. The IFToMM Library of Computational Benchmark Problems [9] is a well-known collection of such examples, which includes test problems for forward- and inverse-dynamics, as well as linearization. These examples illustrate the performance of MBS formulations and implementations when dealing with complex issues such as redundant constraints, singular configurations, stiff problems, and contacts, to mention just a few. Another instance of collection of benchmark problems can be found in [10], which puts forward a series of cases for the validation of flexible multibody dynamics algorithms. Benchmark problems for contact dynamics were introduced in [11]. In the case of MBS dynamics, benchmarking is not limited to the algorithms for the integration of the equations of motion, but has also been extended to applications in which the multibody part is a necessary component, like estimators based on Kalman filters [12]. Benchmark problems for particular applications of MBS dynamics can be found in the areas of railway vehicles [13] and co-simulation [14].

Variable step-size integration methods are frequently used in MBS dynamics applications. These algorithms adapt the step-size of the integration formula to the time-scale of the dynamics of the problem under study with the goal of reducing the time elapsed in computations. In some cases, the step-size control solution is combined with the MBS formulation used to handle the equations of motion, e.g., [15]. This paper introduces a variation of the well-known slider-crank benchmark problem particularly geared towards the assessment of variable step-size integration methods. Two new versions of the linkage were defined, with and without singular configurations. Both were subjected to the action of a force that varied over time, to prevent a periodic system motion.

A particular goal of the present paper is to assess the ability of the variable-step index-3 augmented Lagrangian algorithm with velocity and acceleration projections, introduced in [15], to take advantage of step-size adjustment during motion to deliver more accurate and efficient simulation results. Other formulations and integration formulas were used as well to evaluate the ability of the proposed example to be used in the assessment of variable-step integrators. Results showed that the combination of singular configurations and externally applied forces with variable frequency made the proposed example a challenging problem for most solution methods.

\section{METHODS}

There exist three components that should always be present in the definition of a benchmark problem for MBS dynamics algorithms:

- A definition of the problem to be solved. This must include the specification of the properties and initial state of the mechanical system, as well as information about the manoeuvre to be simulated, such as duration in time and input forces and torques.

- A reference solution which, for the purposes of benchmarking, can be considered correct. This reference solution may be obtained from experimental results, an analytical solution of the problem at hand, or upon convergence of several simulation processes.

- Appropriate error metrics and comparison criteria. These enable the assessment of the solutions obtained with different simulation methods [16].

Moreover, other optional components can be added too, such as reference implementations of the simulation code or data structures to enable the efficient collection and processing of simulation results.

In order to be useful, benchmark examples should be clearly defined problems that are easy to replicate. They should also represent nontrivial scenarios, which are meaningful or challenging in at least one respect. Ideally, they should also be representative of a wider class of systems. For instance, a slider-crank that undergoes singular configurations can be defined in a straightforward way with a reduced set of kinematic and kinetic parameters. In spite of being a relatively 
simple mechanical system, it poses a problem for simulation algorithms that cannot deal with rankdeficient Jacobian matrices; even some solution methods that can handle them need to be carefully adjusted to deliver correct results [5], as is the case of augmented Lagrangian algorithms. Results obtained with this linkage can then be used to assess the general ability of solution methods to carry out the simulation of systems with singularities.

In some cases, it is possible to find an analytical solution for the motion of simple mechanical systems. Generally, this is not the case. In benchmark problems that do not represent a physical system, for which experimental results are not available, physical magnitudes can be used as indicators instead. For instance, the variation of the mechanical energy can be used to quantify the accuracy of a given simulation method if the benchmark problem represents a conservative system. These indicators should be used with precaution, as a precise energy conservation does not necessarily guarantee the correctness of the obtained results. Arriving at a reference solution through the convergence of several simulation methods is advisable when analytical and experimental solutions are not available.

Error metrics and criteria are also an important component of a benchmark problem. First, it is necessary to select the variables that will be selected to evaluate the accuracy of a solution. These may include kinematic variables, such as positions, velocities, and accelerations, or kinetic magnitudes like forces or energies. Usually $n$ variables of interest can be selected and will suffice to measure the precision of the results. It is also necessary to specify at what points in time these variables will be evaluated. When using fixed-step integrators this is relatively simple, as data can be gathered regularly during the simulation. With variable-step simulators usually interpolation methods have to be used. Second, a metric to quantify the deviation of the results obtained with a particular method with respect to the reference solution is necessary. The local error at time point $t_{i}$ for variable $y_{j}$ can be evaluated as

$$
\varepsilon_{j}\left(t_{i}\right)=y_{j}\left(t_{i}\right)-y_{j}^{\mathrm{ref}}\left(t_{i}\right)
$$

where $y_{j}^{\text {ref }}$ denotes the value that corresponds to the reference solution. Relative definitions of the error can be used too [16]. Absolute errors, however, show a better behaviour when the variables of interest approach zero. The total error of a simulation can be calculated as

$$
\varepsilon_{\mathrm{T}}=\sqrt{\frac{1}{n} \sum_{j=1}^{n} \frac{w_{j}}{m} \sum_{i=1}^{m}\left(\varepsilon_{j}\left(t_{i}\right)\right)^{2}}
$$

where $m$ is the total number of time points collected during the simulation and $w_{j}$ is a weight factor that represents the contribution of variable $j$ to the total error. Factor $w_{j}$ can also be used to make errors dimensionless, so that variables with different units can be added together in a single error indicator. Besides the total error in (2), it is also possible to select other indicators, such as the maximum or minimum absolute error for a single variable or group of variables.

The existence of a metric like the one in (2) makes it possible to establish a validity criterion that determines whether a simulation is accurate enough or not. A criterion like this is particularly important if the benchmark problem is to be used to compare several solution approaches in terms of efficiency, because that comparison should be carried out requesting the same accuracy level from every method.

Finally, comparison criteria can be defined to quantify the differences between the different solution methods. The elapsed time in computations is a commonly used criterion to rank algorithms and implementations, but other metrics, such as energy balances and satisfaction of kinematic constraints can be used too [17].

\subsection{Problem description}

The benchmark problem used in this paper is a variation of the well-known slider-crank linkage, already included in the IFToMM benchmark library [9] and shown in Fig. 1. This planar mechan- 
ical system is composed of two rods, links 1 and 2, with uniformly distributed mass $m_{1}$ and $m_{2}$ and length $L_{1}$ and $L_{2}$, respectively. The slider, link 3, has mass $m_{3}$ and moves without friction along the $x$ axis. The mechanism moves under gravity effects with $g=9.81 \mathrm{~m} / \mathrm{s}^{2}$ acting along the negative $y$ axis. The system has one degree of freedom; at time $t=0$, rod 1 is at an angle $\theta_{1,0}$ with respect to the $x$ axis and the velocity of point $\mathrm{Q}$ is $\dot{x}_{\mathrm{Q}, 0}$. A horizontal external force $f$ acts on point $\mathrm{Q}$ during motion.

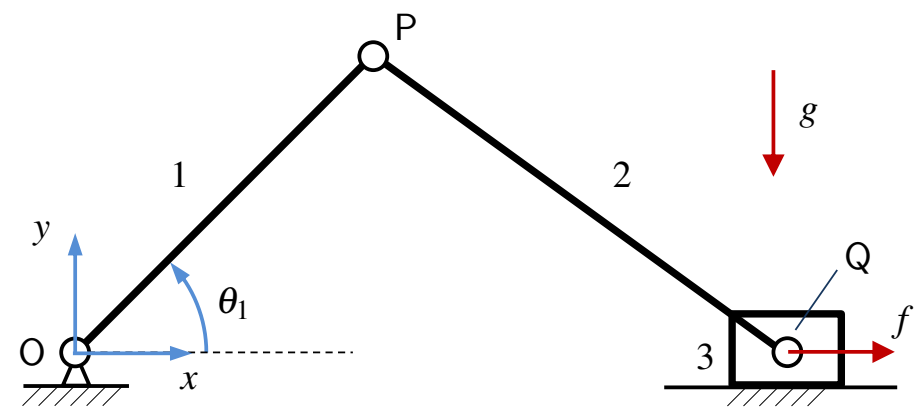

Figure 1. Slider-crank mechanism used as benchmark problem.

Three simulation cases are considered as shown in Table 1, which details the values of the physical parameters of the system, its initial state, and the externally applied actions.

Table 1. Simulation cases

\begin{tabular}{l|cccccccccc} 
Case & $L_{1}$ & $L_{2}$ & $m_{1}$ & $m_{2}$ & $m_{3}$ & $I_{\mathrm{G}, 1}$ & $I_{\mathrm{G}, 2}$ & $\theta_{1,0}$ & $\dot{x}_{\mathrm{Q}, 0}$ & $f$ \\
& $(\mathrm{~m})$ & $(\mathrm{m})$ & $(\mathrm{kg})$ & $(\mathrm{kg})$ & $(\mathrm{kg})$ & $\left(\mathrm{kgm}^{2}\right)$ & $\left(\mathrm{kgm}^{2}\right)$ & $(\mathrm{rad})$ & $(\mathrm{m} / \mathrm{s})$ & $(\mathrm{N})$ \\
\hline $\mathbf{1}$ & 1 & 1 & 1 & 1 & 0 & $1 / 12$ & $1 / 12$ & $\pi / 4$ & -4 & 0 \\
$\mathbf{2}$ & 3 & 6 & 1.5 & 3 & 0.25 & $9 / 8$ & 9 & 0 & 0 & $100 \sin (\pi t)$ \\
$\mathbf{3}$ & 1 & 1 & 1 & 1 & 0 & $1 / 12$ & $1 / 12$ & $\pi / 4$ & 0 & $100 \sin (\pi t)$ \\
\hline
\end{tabular}

Case 1 corresponds to the slider-crank benchmark problem in [9]. In this case, rods 1 and 2 have the same length $\left(L_{1}=L_{2}\right)$ and this causes the linkage to pass through a singular configuration when $\theta_{1}= \pm \pi / 2$, i.e., when both rods are aligned on the $y$ axis. From here the linkage can continue its motion either as a slider-crank mechanism or as a pendulum with point $Q$ motionless at the location of point $\mathrm{O}$. The slider is massless and the externally applied force $f$ in this case is zero during motion.

In case 2, rods 1 and 2 have different lengths and so the linkage motion is not affected by singular configurations. The externally applied force follows now a sinusoidal expression whose frequency increases with time, $f=100 \sin (\pi t)$. The introduction of this force gives rise to numerical difficulties in the solution of the problem. The system ceases to be conservative and its motion is no longer periodic. Moreover, the extreme positions of the motion, reached when both rods are aligned on the $x$ axis and, thus, with the external force $f$, become challenging from the point of view of numerical simulation. There, depending on the value of the force $f$ and the accuracy of the integration process, the motion can continue following one of two possible branches, namely those that correspond to $\dot{\theta}_{1}>0$ and to $\dot{\theta}_{1}<0$.

Case 3 uses the same physical parameters as case 1, but the externally applied force $f$ now follows the sinusoidal expression from case 2. The resulting problem is subjected to both singular configurations and numerical difficulties at the extreme points of the motion. 


\subsubsection{Variables of interest and metrics}

The mechanical system under study has one degree of freedom and a single variable should be enough to keep track of its motion. However, in cases 1 and 3 singular configurations exist and these give rise to the existence of two branches of motion. For this reason, two variables will be monitored and used to evaluate the error in Eq. (2): angle $\theta_{1}$ between link 1 and the $x$ axis, and the $x$ coordinate of point $\mathrm{Q}$ on the slider.

The sampling interval for error evaluation was set to $10 \mathrm{~ms}$; a total simulation length of $10 \mathrm{~s}$ was used in cases 1 and 2, whereas this duration was reduced to $5 \mathrm{~s}$ for case 3 . The total errors associated with variables $\theta_{1}$ and $x_{\mathrm{Q}}$ were aggregated into a single error indicator using Eq. (2) using weights $w_{\theta}=1 \mathrm{rad}^{-2}$ and $w_{x_{Q}}=1 \mathrm{~m}^{-2}$, intended to make the final metric dimensionless. Case 1 is a conservative system, and so the total mechanical energy was used there as additional metric.

When using variable step-size integrators, the variables of interest are usually not evaluated exactly at the sampling points. This also happens when constant integration steps are used, but they are not exact dividers of the sampling interval. In these cases, data need to be interpolated; a linear polynomial interpolation has been used here to determine the necessary values.

Two levels of accuracy have been established for the defined cases. In case 1, a high-precision simulation corresponds to a maximum admissible total error $\varepsilon_{\mathrm{T}}=2 \cdot 10^{-4}$. This is roughly equivalent to the energy criterion set in the IFToMM benchmark, which accepted simulations with maximum energy errors below $0.001 \mathrm{~J}$. The admissibility threshold for low-precision simulation in this case was increased up to $\varepsilon_{\mathrm{T}}=2 \cdot 10^{-3}$. However, different precision requirements can be specified for the three cases; the selected thresholds are shown in Table 2.

Table 2. Maximum admissible errors for each simulation case

\begin{tabular}{l|cc} 
Case & High-precision $\varepsilon_{\mathrm{T}}$ & Low-precision $\varepsilon_{\mathrm{T}}$ \\
\hline $\mathbf{1}$ & $2 \cdot 10^{-4}$ & $2 \cdot 10^{-3}$ \\
$\mathbf{2}$ & $5 \cdot 10^{-3}$ & $5 \cdot 10^{-2}$ \\
$\mathbf{3}$ & $1 \cdot 10^{-2}$ & $1 \cdot 10^{-1}$ \\
\hline
\end{tabular}

The selection of the threshold depends on factors like the time scale of the dynamics and the difficulty of the problem.

\subsection{Solution methods}

Several solution methods were used to carry out the forward-dynamics simulation of the benchmark example. Their main characteristics are summarized on Table 3. Unless otherwise specified, MATLAB implementations were used to perform the simulation.

The first method (AL) uses the index-1 augmented Lagrangian algorithm with position and velocity projections presented in [18], integrated with the trapezoidal rule (TR) in fixed-point iteration form. Method ALi3p stands for the index-3 augmented Lagrangian algorithm with projections of velocities and accelerations $[19,20]$. Algorithm ALi3pvs is the variable-step version of ALi3p introduced in [15]. These three methods described the benchmark problem using a set of 18 natural coordinates [1] subjected to 18 redundant kinematic constraints. Besides, methods mAL and $\mathrm{mNS}$ were also assessed, in which MATLAB ode 45 integration formula is used. The first one uses a stabilized augmented Lagrangian algorithm, while the second adopts a null-space formulation similar to the one in [21]. These methods described the system with Cartesian variables, namely the $x$ and $y$ coordinates of the centre of mass of each body, and impose on them 11 independent constraint equations. In cases 1 and 3, the Jacobian matrix of these constraints loses rank and, as 
Table 3. Summary of the methods employed to solve the slider-crank benchmark problem

\begin{tabular}{l|cccc} 
Method & Coordinates & Constraints & Integrator & Step-size \\
\hline AL & 18 natural, 3D & 18 redundant & TR & fixed \\
ALi3p & 18 natural, 3D & 18 redundant & TR & fixed \\
ALi3pvs & 18 natural, 3D & 18 redundant & TR & variable \\
mAL & 12 Cartesian, 2D & 11 independent & ode45 & variable \\
mNS & 12 Cartesian, 2D & 11 independent & ode45 & variable \\
mAL-ode4 & 12 Cartesian, 2D & 11 independent & ode4 & fixed \\
mNS-ode4 & 12 Cartesian, 2D & 11 independent & ode4 & fixed \\
\hline
\end{tabular}

expected, the mNS method was unable to successfully complete the simulation. For the purpose of comparing fixed- and variable-step integrators, methods mAL and mNS were also combined with a fourth order, fixed-step Runge-Kutta integration formula, denoted in the text as ode4.

\subsection{Reference solution}

Reference solutions for each case were obtained by convergence of the different methods summarized in Section 2.2. An additional simulation with a Simscape model of the mechanism was also included in this process, intended to confirm the results obtained by the authors by means of third-party software. Figures 2 and 3 show the slider displacement $x_{\mathrm{Q}}$ that correspond to the reference solutions of cases 1 and 2. Upon convergence, the differences in the monitored variables across the solutions delivered by the methods remained below $2.5 \cdot 10^{-4} \mathrm{~m}$ for $x_{\mathrm{Q}}$ and $2 \cdot 10^{-4} \mathrm{rad}$ for $\theta_{1}$ at every sampling point. For case 1 , the error in mechanical energy of the reference solution was lower than $2 \cdot 10^{-6} \mathrm{~J}$.

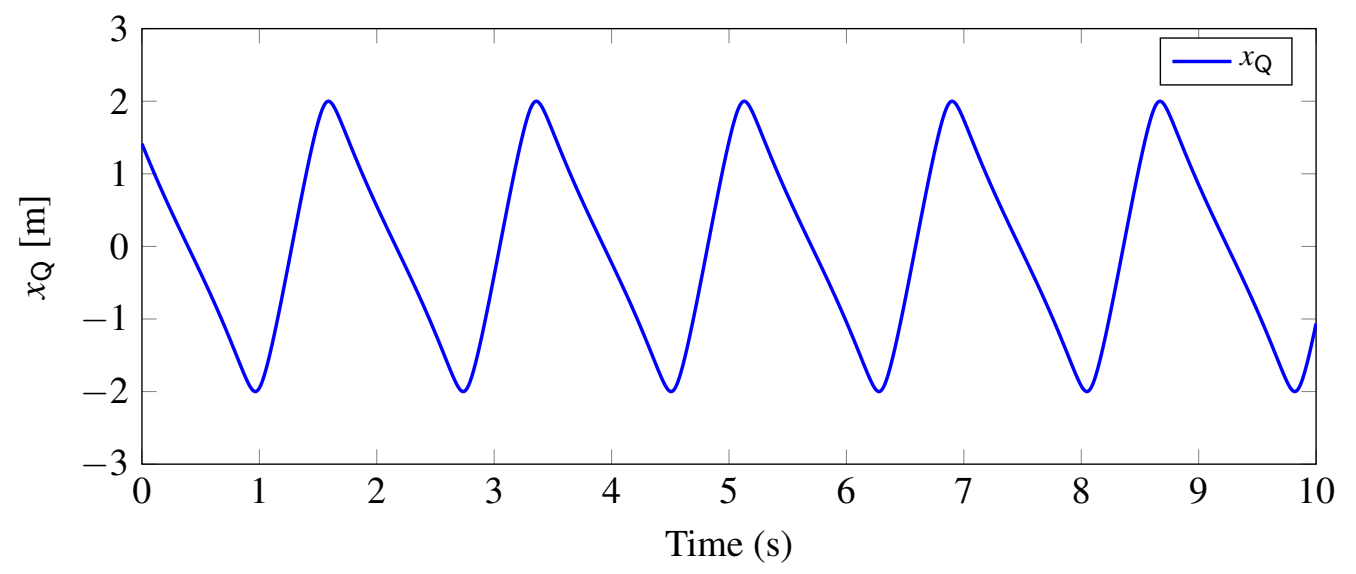

Figure 2. Reference solution: displacement $x_{\mathrm{Q}}$ of the slider in case 1 .

Obtaining a reference solution in case 3 became more complicated. Figure 4 displays the slider displacement delivered by different solution methods in this case; these results show that it was not possible to achieve convergence during the last seconds of the motion, even with very stringent simulation conditions. The system dynamics becomes chaotic due to the combination of singular configurations and the applied force $f$. Under these circumstances, using a $10 \mathrm{~s}$-simulation as benchmark would not convey interesting information regarding the capability of the methods to deliver accurate solutions. For these reasons, the total duration of this numerical experiment was 


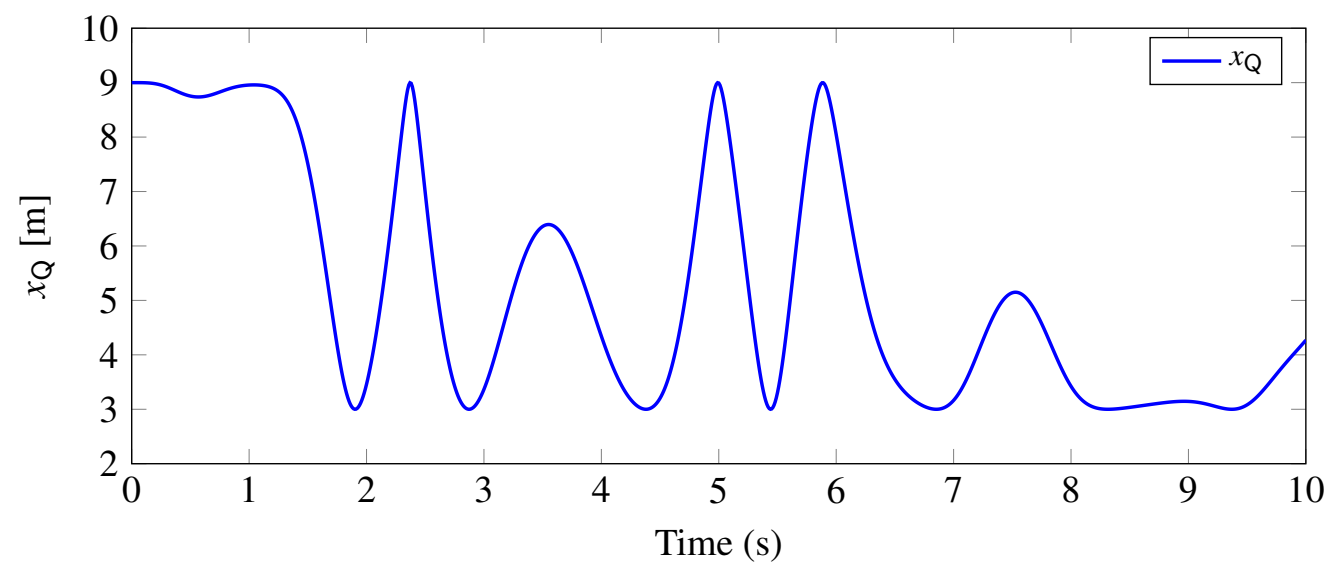

Figure 3. Reference solution: displacement $x_{\mathrm{Q}}$ of the slider in case 2 .

shortened to $5 \mathrm{~s}$. Even with this reduced duration, it was not possible to decrease the maximum differences between solutions at convergence below $7 \cdot 10^{-3} \mathrm{~m}$ for $x_{\mathrm{Q}}$ and $10^{-2} \mathrm{rad}$ for $\theta_{1}$.

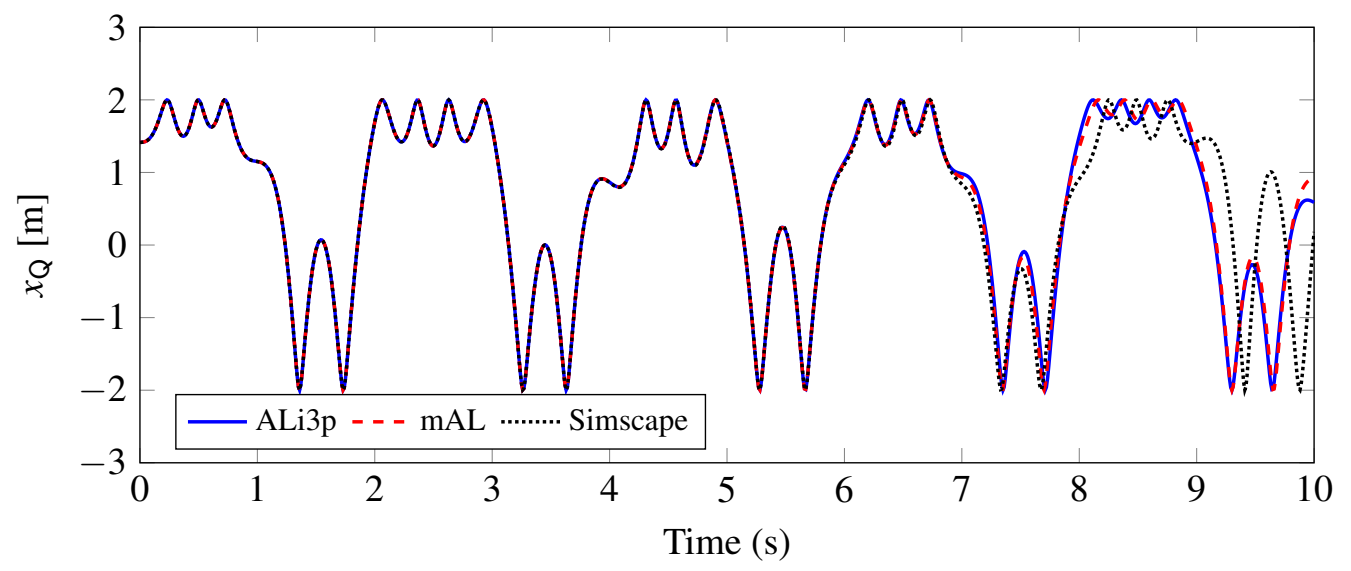

Figure 4. Displacement $x_{\mathrm{Q}}$ of the slider in case 3 obtained with different solution methods.

\section{RESULTS}

The benchmark scenarios defined in Section 2 were used to evaluate and compare the performance of the simulation solutions in Table 3. The computations were performed on an Intel Core i7$7700 \mathrm{HQ}$ at $2.80 \mathrm{GHz}$ with $16 \mathrm{~GB}$ of RAM, running Windows 64-bit and Matlab R2020b.

In the case of the constant-step AL and ALi3p formulations, the integration step-size $h$ was found to be the parameter that had the greatest impact on the efficiency and accuracy of the simulation. Both were able to deal with singular configurations in cases 1 and 3. For the low precision case, both methods delivered comparable results in terms of efficiency. For high precision, the ALi3p method was clearly superior; in case 2 the AL solver was unable to meet the required error threshold. It must be noted that the formulation parameters were the same in all simulation cases, with the notable exception that case 3 required the use of a higher penalty factor in the ALi3p solution ( $\alpha=10^{15}$ instead of $\alpha=10^{12}$ used in the other two cases). The results delivered by ALi3p were used next for comparison with those delivered by its variable-step counterpart. A summary of the results of the most efficient simulations for high and low precision levels is shown in Table 4. The elapsed time corresponds to the average of three runs of each scenario.

For the variable-step ALi3pVS method, attaining the levels of accuracy specified in Table 2 de- 
Table 4. Best results obtained with the ALi3p solver

\begin{tabular}{c|ccc|ccc|} 
& \multicolumn{3}{|c}{ High precision } & \multicolumn{3}{c}{ Low precision } \\
& $\varepsilon_{\mathrm{T}}$ & Elapsed $(\mathrm{s})$ & $h(\mathrm{~ms})$ & $\varepsilon_{\mathrm{T}}$ & Elapsed $(\mathrm{s})$ & $h(\mathrm{~ms})$ \\
\hline Case 1 & $1.6 \cdot 10^{-4}$ & 11.07 & 1.5 & $1.9 \cdot 10^{-3}$ & 4.82 & 3.5 \\
Case 2 & $3.6 \cdot 10^{-3}$ & 17.83 & 0.75 & $4.8 \cdot 10^{-2}$ & 5.31 & 3 \\
Case 3 & $5.4 \cdot 10^{-3}$ & 24.83 & 0.25 & $8.7 \cdot 10^{-2}$ & 3.95 & 2.25 \\
\hline
\end{tabular}

pended on a number of factors. The user must specify the upper and lower limits within which the integration step $h$ has to remain, $h_{\max }$ and $h_{\min }$. Besides these, the criteria used to stop the NewtonRaphson iteration, namely the number of iterations $\gamma$ and the norm of the maximum admissible increment of the variables upon convergence, $\varphi_{\max }$, were found to be the most relevant. Tuning these parameters was not a straightforward process, because the impact of a given selection on the simulation performance is highly nonlinear. Moreover, because the algorithm adjusts the step-size based on its previously used value, the initially used step-size $h_{0}$ must also be considered a parameter of the simulation. In the reported simulations, these parameters were varied in the following ranges: $h_{\max } \in[0.5,10] \mathrm{ms}, h_{\min } \in[0.05,1] \mathrm{ms}, \gamma \in[1,10], \varphi_{\max } \in\left[10^{-5}, 10^{-15}\right]$. Table 5 shows the parameter combinations that delivered the most efficient simulation for the high-precision requirement in every case. In case 3 it was not possible to achieve an improvement over the constant-step ALi3p method.

Table 5. Best results obtained with the ALi3pvs solver, high precision

\begin{tabular}{c|ccccccc} 
& $\varepsilon_{\mathrm{T}}$ & Elapsed $(\mathrm{s})$ & $h_{\max }(\mathrm{ms})$ & $h_{\min }(\mathrm{ms})$ & $h_{0}(\mathrm{~ms})$ & $\gamma$ & $\varphi_{\max }$ \\
\hline Case 1 & $9.1 \cdot 10^{-5}$ & 9.2 & 10 & 0.05 & 0.5 & 10 & $10^{-7}$ \\
Case 2 & $2.8 \cdot 10^{-3}$ & 17.7 & 5 & 0.05 & 1 & 2 & $10^{-11}$ \\
Case 3 & Same as ALi3p & - & - & - & - & - \\
\hline
\end{tabular}

Table 6 shows the results delivered by the step-size adjustment method for the low-precision case.

Table 6. Best results obtained with the ALi3pvs solver, low precision

\begin{tabular}{c|ccccccc} 
& $\varepsilon_{\mathrm{T}}$ & Elapsed $(\mathrm{s})$ & $h_{\max }(\mathrm{ms})$ & $h_{\min }(\mathrm{ms})$ & $h_{0}(\mathrm{~ms})$ & $\gamma$ & $\varphi_{\max }$ \\
\hline Case 1 & $1.7 \cdot 10^{-3}$ & 3.3 & 3.5 & 0.05 & 2 & 2 & $10^{-6}$ \\
Case 2 & $1.1 \cdot 10^{-2}$ & 3.7 & 10 & 0.05 & 1 & 2 & $10^{-7}$ \\
Case 3 & $9.9 \cdot 10^{-2}$ & 3.68 & 2 & 0.05 & 1 & 2 & $5 \cdot 10^{-8}$ \\
\hline
\end{tabular}

It is difficult to provide general recommendations on the selection of the ALi3pvs parameters. For low-precision simulations, it seems advisable to decrease the limit of admissible iterations per step $\gamma$ and regulate the error of the simulation by tuning the convergence criterion $\varphi_{\max }$.

The $\mathrm{mAL}$ and $\mathrm{mNS}$ solvers completed the simulation of the example in shorter times than the AL, ALi3p, and ALi3pvs methods. This is explained by the different modelling used, which led to a smaller problem size, and also by the fact that the code was specifically implemented to deal with this example, while the former methods employed a library that took care of the automatic generation of the equations of motion. The purpose of this Section, however, is not comparing 
the different methods, but verifying the effect of using variable-step integration to perform the simulation.

As mentioned, the mNS solver was unable to deal with the singular configurations in cases 1 and 3. In case 2, nonetheless, it delivered the most efficient solution. The results obtained in this case with mNS were compared to a fixed-step counterpart of the method that used a fourth order Runge-Kutta formula with a constant step-size (ode4). This comparison is shown in Fig. 5; data from $\mathrm{mAL}$ are included as well. These results show that variable-step solvers were able to increase the precision of the computations while keeping the required computational load lower than their fixed-step counterparts.

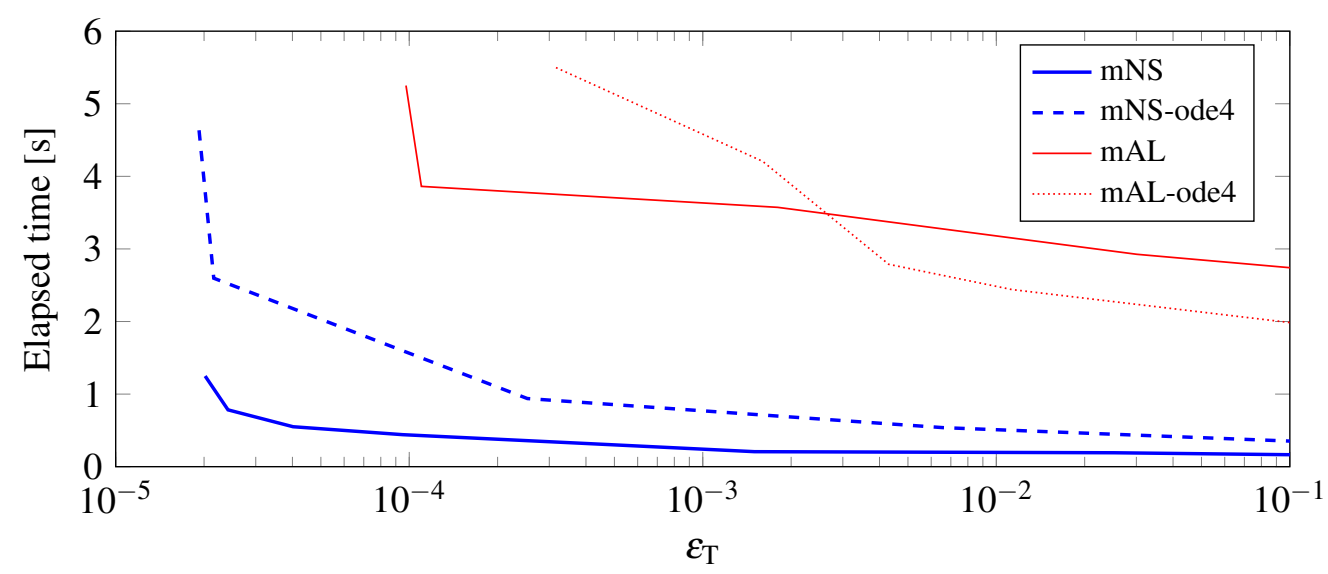

Figure 5. Elapsed time in the solution of case 2 as a function of the precision $\varepsilon_{\mathrm{T}}$ delivered by each method.

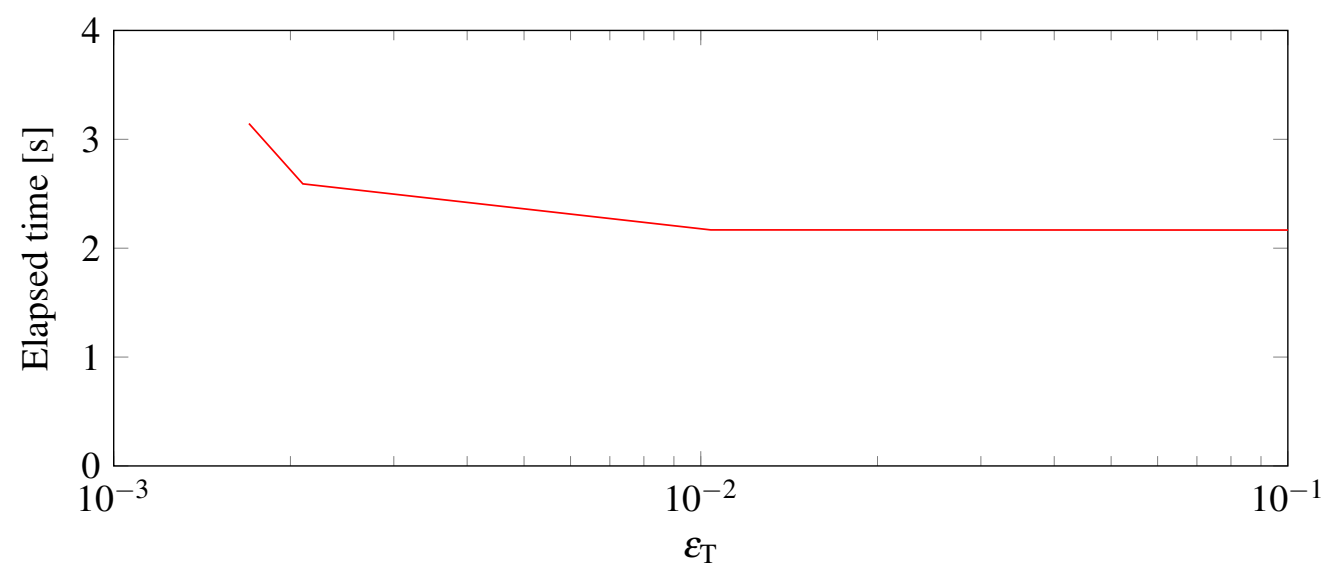

Figure 6. Elapsed time in the solution of case 3 as a function of the precision $\varepsilon_{\mathrm{T}}$ delivered by method mAL.

The results in case 1 for methods mAL and mAL-ode4 followed similar trends to those shown in Fig. 5: when higher precision was required, variable-step integration showed a comparative advantage with respect to the fixed-step method. In case 3 the mAL-ode4 integration only managed to converge to the reference solution with step-sizes below $1.5 \mathrm{~ms}$; moreover, decreasing the integration step-size did not improve the accuracy of the results. In this case, the variable-step method delivered better results for all precision levels; these are summarized in Fig. 6. 


\section{CONCLUSIONS}

In this work, a benchmark example for the evaluation of variable-step multibody dynamics formulations has been introduced. The selected example consists in three variations of a planar slider-crank linkage, with different physical parameters and applied external forces with variable frequency. The combination of these forces with the existence of singular configurations in the mechanism resulted in a challenging problem for variable-step integration methods. A common approach used by these methods is the reduction of the step-size when it is necessary to deal with fast dynamics; this poses a problem if the mechanism is moving near a singular configuration, because the step-size reduction often leads to numerical problems in these positions.

The slider-crank linkage example also served to highlight relevant aspects of the definition of benchmark problems, such as the availability of a reference solution and the necessity of suitable metrics to compare the performance of different solution methods.

The three proposed cases of this benchmark problem were used to assess the ability of variablestep integration methods for multibody system dynamics to improve the efficiency delivered by their fixed-step counterparts. Results showed that the performance of each approach depends on the precision requirements and the characteristics of the problem being solved and confirmed the usefulness of the proposed example for benchmarking applications.

\section{ACKNOWLEDGMENTS}

F. González acknowledges the support of the Ministry of Economy of Spain through the Ramón y Cajal programme, contract RYC-2016-20222.

\section{REFERENCES}

[1] García de Jalón, J., Bayo, E.: Kinematic and Dynamic Simulation of Multibody Systems: The Real-Time Challenge. Springer-Verlag, New York (NY), USA (1994) ISBN:3-54094096-0.

[2] Bauchau, O.A.: Flexible Multibody Dynamics. Springer, Dordrecht, The Netherlands (2011) ISBN:978-94-007-0334-6.

[3] Featherstone, R.: Robot dynamics algorithms. Springer, New York (NY), USA (1987) ISBN:978-1-4757-6437-6.

[4] García de Jalón, J., Gutiérrez-López, M.D.: Multibody dynamics with redundant constraints and singular mass matrix: existence, uniqueness, and determination of solutions for accelerations and constraint forces. Multibody System Dynamics 30(3) (2013) 311-341

[5] González, F., Dopico, D., Pastorino, R., Cuadrado, J.: Behaviour of augmented Lagrangian and Hamiltonian methods for multibody dynamics in the proximity of singular configurations. Nonlinear Dynamics 85(3) (2016) 1491-1508

[6] González, M., González, F., Dopico, D., Luaces, A.: On the effect of linear algebra implementations in real-time multibody system dynamics. Computational Mechanics 41(4) (2008) $607-615$

[7] Torres-Moreno, J.L., Blanco-Claraco, J.L., López-Martínez, J., Giménez-Fernández, A.: A comparison of algorithms for sparse matrix factoring and variable reordering aimed at realtime multibody dynamic simulation. In: Proceedings of the ECCOMAS Thematic Conference on Multibody Dynamics, Zagreb, Croatia (2013)

[8] González, M., González, F., Luaces, A., Cuadrado, J.: A collaborative benchmarking framework for multibody system dynamics. Engineering with Computers 26(1) (2010) 1-9 
[9] IFToMM Technical Committee for Multibody Dynamics: Library of computational benchmark problems (2021) URL:https://www.iftomm-multibody.org/benchmark/.

[10] Bauchau, O.A., Betsch, P., Cardona, A., Gerstmayr, J., Jonker, B., Masarati, P., Sonneville, V.: Validation of flexible multibody dynamics beam formulations using benchmark problems. Multibody System Dynamics 37(1) (2016) 29-48

[11] Masoudi, R., Flores, P., McPhee, J.: Benchmark problems for contact dynamics in multibody systems. In: The 3rd International Conference on Multibody System Dynamics (IMSD), Busan, South Korea (2014)

[12] Sanjurjo, E., Naya, M.Á., Blanco-Claraco, J.L., Torres-Moreno, J.L., Giménez-Fernández, A.: Accuracy and efficiency comparison of various nonlinear Kalman filters applied to multibody models. Nonlinear Dynamics 88(3) (2017) 1935-1951

[13] Bezin, Y., Pålsson, B.A.: Multibody simulation benchmark for dynamic vehicle-track interaction in switches and crossings: modelling description and simulation tasks. Vehicle System Dynamics (2021) 1-16

[14] Zar, A., González, F., Rodríguez, B., Luaces, A., Naya, M.A., Cuadrado, J.: Benchmark problems for co-simulation methods. In: COSIM 2021: International Symposium on CoSimulation and Solver Coupling in Dynamics, Ferrol, Spain (2021)

[15] Dopico, D., Sanjurjo, E., Cuadrado, J., Luaces., A.: A variable time-step and variable penalty method for the index-3 augmented Lagrangian formulation with velocity and acceleration projections. In: Proceedings of the ECCOMAS Thematic Conference on Multibody Dynamics, Prague, Czech Republic (2017)

[16] González, M., Dopico, D., Lugrís, U., Cuadrado, J.: A benchmarking system for MBS simulation software: Problem standardization and performance measurement. Multibody System Dynamics 16(2) (2006) 179-190

[17] Marques, F., Souto, A.P., Flores, P.: On the constraints violation in forward dynamics of multibody systems. Multibody System Dynamics 39(4) (2016) 385-419

[18] Bayo, E., Ledesma, R.: Augmented Lagrangian and mass-orthogonal projection methods for constrained multibody dynamics. Nonlinear Dynamics 9(1-2) (1996) 113-130

[19] Cuadrado, J., Cardenal, J., Morer, P., Bayo, E.: Intelligent simulation of multibody dynamics: space-state and descriptor methods in sequential and parallel computing environments. Multibody System Dynamics 4(1) (2000) 55-73

[20] Dopico, D., González, F., Cuadrado, J., Kövecses, J.: Determination of holonomic and nonholonomic constraint reactions in an index-3 augmented Lagrangian formulation with velocity and acceleration projections. Journal of Computational and Nonlinear Dynamics 9(4) (2014)

[21] Shabana, A.A.: Computational Dynamics. Second edn. Wiley, Hoboken, NJ, USA (2001) ISBN:978-0-471-05326-2. 